\title{
Assessments of Therapeutic Effects of Platelet-rich Plasma in Knee Osteoarthritis: Possible Role of Inflammatory Cytokines
}

Mohammed H. Hassan ( $\sim$ mohammedhosnyhassaan@yahoo.com )

Associate Professor of Medical Biochemistry, Faculty of Medicine, South Valley University, Qena 83523, Egypt. https://orcid.org/0000-0003-2698-9438

\section{Tahia H. Saleem}

Assiut University Faculty of Medicine

\section{Sawsan Abuhamdah}

Al Ain University

\section{Hamdy Tammam}

South valley University

Nehal Ashraf Zaki

South valley University

\section{Ghada M. Abdel-Razik}

South Valley University

\section{Safaa Y. Saleem}

Assiut University Faculty of Medicine

\section{Elsayed Said}

South Valley University

\section{Research article}

Keywords: Platelet rich plasma, Knee osteoarthritis, MRI Osteoarthritis Knee Score, pain score, synovial fluid, tumor necrosis factor-a, macrophage migration inhibitory factor

Posted Date: September 8th, 2020

DOl: https://doi.org/10.21203/rs.3.rs-63573/v1

License: (c) (i) This work is licensed under a Creative Commons Attribution 4.0 International License. Read Full License

Version of Record: A version of this preprint was published on June 15th, 2021. See the published version at https://doi.org/10.3844/ajbbsp.2021.267.280. 


\section{Abstract}

Background: Osteoarthritis $(\mathrm{OA})$ is a multifactorial disease that commonly affects the knee. Tumor necrosis factor- $a$ (TNF- $a$ ) is able to regulate inflammation in OA. Macrophage migration inhibitory factor (MIF) may be involved in the pathophysiology of arthritis. Platelet-rich plasma (PRP) may reduce pain associated with OA. The current study aimed to assess the possible therapeutic effects of PRP in patients with knee OA of various severities.

Methods: A prospective study was performed on 90 patients were included and categorized into mild (30 cases, moderate (30 cases) and severe (30 cases) knee OA. Three intra-articular (I.A) injections of PRP, 2 weeks a part, were received. Pain score and MRI Osteoarthritis Knee Score (MOAKS) were assessed. Serial synovial fluid cytokines assays in the form of Tumor necrosis factor-a (TNF-a) and Macrophage migration inhibitory factor (MIF), were performed using commercially available ELISA assay kits. The assays were performed pre-injection (S1), two weeks from the $1^{\text {st }}$ I.A injection and two weeks from the $2^{\text {nd }}$ I.A injection (S3) for all included patients.

Results: The mean values of pain score and synovial TNF-a and MIF levels were significantly higher

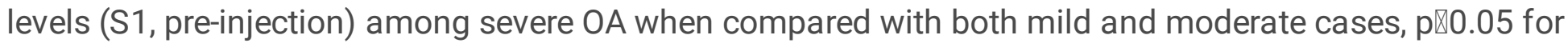
all. There were significantly lower pain score and synovial TNF-a and MIF levels at S3 in mild, moderate and severe knee OA when compared with S1 values, $\mathrm{p} \otimes 0.05$ for all. There was significant improvement in synovitis in both mild and moderate cases ( $\mathrm{p} \otimes 0.05$ for both).

Conclusion: I.A injection of PRP significantly reduces the synovial fluid TNF-a and MIF levels with great therapeutic effects on both synovitis via reducing inflammatory cytokines, and bone marrow lesions mainly for mild knee OA and to a lesser extent for moderate cases.

\section{Background}

Osteoarthritis (OA) is a disease primarily affecting the knee that is defined as a progressive loss of joint function and pain from a gradual deterioration of the articular cartilage [1]. Unfortunately, it is a frequent site due to the high use and stress of the knee joint for painful conditions including OA [2].

OA was thought to be primarily a cartilage degenerative disease; however, the latest research has shown that $\mathrm{OA}$ is a multifactorial cause, involving numerous causative factors such as trauma, mechanical forces, inflammation, biochemical reactions, and metabolic derangements [3]. The role of inflammation is not well understood and there is an ongoing debate to determine whether the inflammatory reaction triggers changes in the $\mathrm{OA}$ or, rather, the inflammation is secondary to changes in the $\mathrm{OA}[3]$.

The tumor necrosis factor (TNF) a has been shown to be associated with the progression of osteoarthritis. It has been shown that TNF-a is capable of regulating inflammation in an osteoarthritis rat model by downregulating the signaling pathway of phosphoinositide 3-kinase / protein kinase B (PI3K / AKT) in synovial fibroblasts [4]. 
Macrophage migration inhibitory factor (MIF) is a pro-inflammatory cytokine produced by macrophages which may contribute to arthritis pathophysiology by promoting inflammation and angiogenesis [5].

Recently, current approaches to OA treatment have integrated the use of biologics which mediate the inflammatory process [1]. Platelet-rich plasma (PRP) is increasingly being used for its ability to influence tissue regulation due to growth factors in high platelet levels, thereby reducing OA-related pain [6, 7]. PRP is obtained after whole blood centrifugation, yielding a highly concentrated product with platelets. The agranules within the concentrated platelet solution contain growth factors and proteins that are vital to the coagulation cascade [8]. PRP is thought to influence degeneration of cartilage by altering the autophagy in chondrocytes. Aging cartilage gradually loses its reversible quiescence and its ability to self-renew [9].

The current work aimed to use clinical and radiological scores to determine the potential therapeutic effects of PRP in patients with various severities of knee OA. In addition, serial biochemical assays of the TNF-a and MIF levels of synovial fluid and their associations with both pain and radiological scores were performed among these patients.

\section{Materials And Methods}

\section{Study design and participants}

The present prospective study was conducted on 90 patients with knee OA, recruited from the orthopedic outpatient clinics, Qena University Hospital- South Valley University- Egypt, during the period from February, 1st 2019 to January, 31st 2020. Based on radiological assessments, the included patients were categorized into three groups (mild, moderate and severe $\mathrm{OA}$ ) according to the OA severity. Each group included 30 patients.

The exclusion criteria is as follows: polyarticular disease; knee arthroscopy in the previous year; HA or steroid IA penetration in the preceding 3 months; history of infectious disease and autoimmune disorders such as diabetes, rheumatoid arthritis, hematologic diseases (coagulopathy), serious cardiovascular diseases, infections or immunodepression; anticoagulant therapy or an anti-aggregating agent; use of non-steroidal anti-inflammatory drugs 2 weeks prior to blood sampling; and $<10 \mathrm{~g} / \mathrm{dL}$ of hemoglobin [10].

\section{Clinical assessments}

Detailed medical history and thorough clinical examination from every included subject involving age, gender, weight, height, calculation of body mass index (BMI) $\left(\mathrm{kg} / \mathrm{m}^{2}\right)$, were recorded. As regards the BMl, it is classified into: underweight (if BMI 118.5 ), normal weight (18.5-24.9), overweight (25-29.9), obesity class 1 (30-34.9), obesity class 2 (35-39.9), extreme obesity class 3 ( $(40)$ [11]. Local examination of both knee joints and assessment of knee joint pain for the included patients was done using Visual Analog Scale (VAS) score [12]. OA diagnosis was based on visual identification of the presence of eburning on the articular surfaces of the distal femur, proximal tibia, or patella, right or left. Eburnation is a sclerotic, 
ivory-like subchondral bone reaction that takes place from bone-on-bone contact at sites exposed to advanced cartilage erosion[13.14]. The VAS is a common tool that uses a $10 \mathrm{~cm}$ scale for pain intensity measurement, where $0=$ no pain and $10=$ unable to move. Pain score assessment was performed before beginning PRP therapy and two weeks after the second PRP IA injection.

\section{Radiological assessments of severity of knee OA}

MRI Osteoarthritis Knee Score (MOAKS) was used to assess the severity of knee OA [15]. In brief, for assessment of both bone marrow abnormality or patella-femoral cartilage volume (grade I (mild if the lesion involves $₫ 33 \%$ of subregional volume); grade II (Moderate: if the lesion involves $33 \%-66 \%$ of subregional volume), and grade III (severe: if the lesion involves $₫ 66 \%$ of subregional volume).Regarding synovitis: grade 1 (mild or small - fluid continuous in the retropatellar space); grade 2 (Moderate or medium - slight convexity of the suprapatellar bursa), and grade 3 (severe or large - evidence of capsular distension ). Meniscal desintegrity score of $0-3$ applied for amount of extrusion in 4 locations: medial meniscus (medial and anterior extrusion) and lateral meniscus (medial and anterior extrusion).

Biochemical workup

A. PRP preparation: The PRP needed for IA injection has been prepared under complete aseptic conditions. In order to avoid the effect of food intake on purified PRP, on the day of injection the patients were instructed to fast for 4 hours before blood collection. Approximately $20 \mathrm{~mL}$ of venous blood were drawn from the antecubital vein using an aseptic technique in an effort to avoid irritation and trauma to the platelets. The blood was obtained as an anticoagulant in ten extraction tubes ( $2 \mathrm{~mL}$ each), containing sodium citrate. The tubes were then centrifuged at $2100 \mathrm{rpm}$ at room temperature for 8 minutes to separate the blood in each tube into the plasma, the buffy coat and the residual red blood cells (RBCs) [10]. Using a pipette, the PRP situated just above the selectively precipitated RBCs but not including the buffy coat was carefully aspirated from each tube. In each affected joint, 10-mL PRP samples (from the 10 blood-collecting tubes) were obtained for each patient, and $5 \mathrm{~mL}$ were used for IA injection [11].

B. Serial assays of synovial fluid pro-inflammatory cytokines (TNF- $a$ and MIF): TNF-a and MIF were measured in the synovial fluid samples, using commercially available ELISA assay kits supplied by Chongqing biospes, China, Catalog No: BYEK3327-48T, and BYEK3015, respectively, using microplate ELISA reader (EMR 500, USA), according to manufacture protocol. To extract cell debris, synovial fluid samples were immediately centrifuged at $3000 \mathrm{rpm}$ for $15 \mathrm{~min}$, and the supernatant was aliquoted into $1-\mathrm{mL}$ cryotubes and frozen at $-80^{\circ} \mathrm{C}$ until use. The serial assays were performed to the synovial fluid samples three times. First sample (S1) was pre-injection; the second sample (S2) was two weeks from the first injection, and the third sample (S3) was two weeks from the second injection.

\section{Procedure and timing of IA injections of PRP}


The patient was put in 20 degree flexion in supine position with the knee. Under aseptic conditions, a 21gage needle was used to inject $5 \mathrm{~mL}$ of PRP into the suprapatellar knee joint pouch, using a superolateral method. Local anesthetics were not used. Patients were instructed after the injection to refrain from physical exercise for at least 24 hours but no restriction was specified regarding activities of daily living. Three IA-PRP injections were administered at 2-week intervals. The same physician who was engaged in selecting and testing participants did the injections [10].

\section{Statistical analysis}

IBM SPSS Statistics v.22 was used for analysis of the data. Mean \pm SD was used for expression of quantitative data, while, number and percentage were used for qualitative data. Kolmogro-Smirnov and Shapiro-Wilk normality tests were used. Mann-Whitney $U$ test was used when comparing between two quantitative data and one way-ANOVA was for comparison between more than two quantitative data. Post Hoc test was used for multiple comparisons between different variables. For qualitative variables, Chi-square ( $\chi 2)$ and Fisher's Exact tests were used. Pearson's correlation coefficient $(r)$ : test was used for correlating data. The level of significance was considered $P$ value $₫ 0.05$.

\section{Results}

\section{Demographic and clinical characteristics of the study participants}

The current study included 90 patients with knee OA (9 males (10\%) and 81 (90\%) females), with female: male ratio $9: 1$. Their mean age was 45.5 years $\pm 10.6 \mathrm{SD}$ with age range of 25 to of 65 years. Their mean BMI was $31.9 \pm 2.9 \mathrm{~kg} / \mathrm{m}^{2}$ with range from 23 to $37 \mathrm{~kg} / \mathrm{m}^{2}$. The mean duration of knee OA was $28.8 \pm$ 17.5 months with range from 3 to 60 months. According to the BMI, 7 cases (7.7\%) were categorized as overweight, 69 cases $(76.6 \%)$ were categorized as obesity class 1 , while obesity class 2 was presented in 14 cases $(15.5 \%)$.

All patients have unilateral knee $O A$. Regarding to the severity of $O A$, the included patients were categorized into three groups: 30 cases ( 6 males and 24 females) have mild knee OA (33.3\%), 30 patients (30 females) with moderate severity (33.3\%) and 30 (3 males and 27 females) cases (33.3\%) have severe degree of knee OA. Regarding these groups, there were non-significant differences as regards their mean age $(46.8 \pm 10.2,46 \pm 12.3$, and $43.6 \pm 10.1$ years old, respectively) or BMI $(31 \pm 2.4,32.1 \pm 2.1$, and $32.6 \pm$ 3.9 , respectively) or sex, $\mathrm{p} \otimes 0.05$ for all.

Serial synovial fluid levels of TNF-a as regards time of intra-articular PRP injection and severity of knee $\mathrm{OA}$

As regards the mean values of synovial TNF-a levels $(\mathrm{pg} / \mathrm{mL}$ ), there were significantly higher levels ( $\mathrm{S} 1$, pre-injection) among patients with severe OA $(144.8 \pm 13.6)$ when compared with both mild $(35.5 \pm 7.4)$ and moderate $(97.3 \pm 31.7)$ cases, $\mathrm{p} \otimes 0.05$ for all (Fig. 1A). There were significantly lower synovial TNF-a 
levels two weeks after the second I.A injection of PRP (S3) in patients with mild or moderate or severe knee $\mathrm{OA}(9.3 \pm 5.4,44.8 \pm 11.5$, and $59.7 \pm 3.9$, respectively) when compared with both the pre-injection synovial fluid levels ( $35.5 \pm 7.4,97.3 \pm 31.7$, and $144.8 \pm 13.6$, respectively) and two weeks from the 1 st injection (S2) levels $(17.8 \pm 7.5,64.6 \pm 15.3$, and $82.4 \pm 4.3$, respectively), $\mathrm{p} \otimes 0.05$ for all (Table.1).

Regarding to the mean values of synovial MIF levels $(\mu \mathrm{g} / \mathrm{L})$, there were significantly higher levels (S1, preinjection) among patients with severe $O A(315.1 \pm 23.2)$ when compared with both mild $(7.7 \pm 1.5)$ and moderate ( $47.8 \pm 14.9)$ cases, $\mathrm{p} \otimes 0.05$ for all (Fig. 1B). There were significantly lower synovial TNF-a levels two weeks after the second I.A injection of PRP (S3) in patients with mild or moderate or severe knee $\mathrm{OA}(1.25 \pm 0.9,10.6 \pm 2.6$, and $82.9 \pm 8.2$, respectively) when compared with the pre-injection synovial fluid levels $(7.7 \pm 1.5,47.8 \pm 14.9$, and $315.1 \pm 23.2$, respectively) and two weeks from the 1 st injection (S2) levels $(4.9 \pm 0.9,25.3 \pm 7.9$, and $107.7 \pm 12.3$, respectively), $\mathrm{p} \otimes 0.05$ for all (Table.2).

There were significantly positive correlations between the synovial fluid TNF-a and MIF levels in S1 ( $r=$ $0.815, p \otimes 0.05)$, S2 ( $r=0.783, p \otimes 0.05)$, and S3 $(r=0.750, p \otimes 0.05)$.

\section{Effect of I.A injection of PRP on pain score among patients with knee osteoarthritis}

There were significantly lower mean pain score values when assessed two weeks from the second I.A injection in patients with mild, moderate or severe knee $O A(1.6 \pm 0.5,2.4 \pm 1.9$, and $5.1 \pm 1.9$, respectively) vs. the pre-injection pain score (3.1 $\pm 0.9,5.9 \pm 0.9$, and $8.7 \pm 0.7$, respectively), p $₫ 0.05$ for all (Table.3).

\section{Therapeutic effects of I.A injection of PRP on MOAKS in patients with knee OA}

Comparing MOAKS pre-injection vs. two weeks from the 2nd I.A injection of PRP, there was significant improvement in the bone marrow lesions among patients with mild knee OA only (pष0.05), with significant improvement in the synovitis in both patients with mild or moderate knee $0 A$ ( $\mathrm{p} \otimes 0.05$ for both), (Fig. 2A and B, Table.4).

There were no significant improvements in both patello-femoral cartilage volume and meniscal desintegrity in various severities of knee OA, $\mathrm{p} \otimes 0.05$ for all (Fig. 2C, Table.4).

\section{Correlations of pain score with serial synovial TNF- $a$ and MIF levels}

Among the included patients with knee $O A$, there were significantly positive correlations between preinjection synovial fluid TNF-a levels (S1) with the pre-injection pain score (0.84, p $₫ 0.05)$ ), (Fig. 3A), and between synovial fluid TNF-a levels 2 weeks after the 2 nd injection (S3) and the post-injection pain score

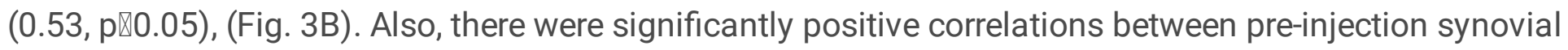
fluid MIF levels (S1) with the pre-injection pain score (0.88, pष0.05), (Fig. 4A), and between synovial fluid

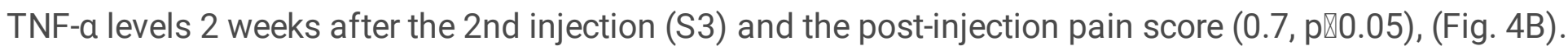




\section{Therapeutic outcomes of the IA injection of the PRP in patients with various severities of knee OA}

Comparing the therapeutic efficiency of I.A injection of PRP among patients with knee OA, there was improvement in all patients with mild knee OA (30 cases, 100\%), while 15 cases (50\%) with knee OA showed improvement and only 6 cases $(20 \%)$ of patients with severe knee OA showed improvement regarding pain score (Table.5).

\section{Discussion}

Knee osteoarthritis $(\mathrm{OA})$ in subjects over 50 years of age is a major cause of pain and impairment with a serious effect on physical activity and quality of life [16]. The present study evaluates the possible therapeutic effects of I.A injection of PRP in patients with knee OA using pain score, MOAKS and biochemical serial assays of synovial fluid cytokines levels in the form of TNF-a and MIF .

Obesity and overweight have long been recognized as important risk factors for $\mathrm{OA}$, in particular knee $\mathrm{OA}$ [17]. The current study revealed female predominance with female: male ratio 9:1. Also, there was higher frequency for patients categorized as obesity class 1 followed by obesity class 2 , while those categorized as overweight have the least frequency among patients with knee OA. These were corresponds with the findings of previous studies [18-23]. The cause of female predominance may be multifactorial and these involve structural variations, prior trauma, genetic and hormonal disorders, as men had significantly higher total tibial and patella cartilage volumes than women and women had a significantly higher prevalence of baseline patellar cartilage defects. Over time women showed more volume loss in the knee cartilage compared to their male counterparts [24]. The key but not only mechanism by which obesity will lead to knee OA is possibly increased mechanical loading on the joint. Knee overloading may cause synovial joint breakdown and failure of ligament and other structural supports [24].

While platelet-rich plasma (PRP) has been approved as an agent for knee osteoarthritis therapy, different studies of PRP in the OA knee have found more consistently positive results compared to hyaluronic acid, other intraarticular injections and placebo than in other musculoskeletal tissues [25, 26]. Although the causes of osteoarthritis in the knee are not fully understood, laboratory and clinical evidence suggest that inflammatory cytokines can contribute to its pathogenesis $[27,28]$. The present study revealed significantly higher synovial fluid inflammatory cytokines (TNF-a and MIF) levels (S1, pre-injection) among patients with severe OA when compared with both mild and moderate cases with significantly lower synovial TNF-a and MIF levels two weeks after the second I.A injection of PRP (S3) in patients with mild or moderate or severe knee OA when compared with both the pre-injection synovial fluid levels and two weeks from the 1st injection (S2) levels. These indicate that PRP treatment for patients with knee osteoarthritis had beneficial effects in regulating inflammatory factors. Inflammatory cytokines have been documented to form a complex regulatory signal network in femoral head osteonecrosis which is mediated by different intracellular kinase signaling pathways to regulate the recruitment, stimulation and activation of autoimmune cells [29]. In accordance, Zhang et al [30] reported that MIF levels in synovial fluid were independently associated with the severity of self-reported pain in OA patients but not in serum. 
Huang et al [25] reported significantly down-regulated plasma concentrations of cytokines involving TNFa after PRP treatment in patients with knee OA.

The current study reported significantly positive correlations between the synovial fluid TNF- $a$ and MIF levels among the included patients with knee OA. MIF is a potent pro-inflammatory cytokine that can cause the release of many inflammatory cytokines such as interferon (IFN)- $y$, interleukin (IL)-1ß, 6, 8 and TNF-a by initiating an inflammatory cascade [31].

Regarding the assessments of the therapeutic effects of I.A injection of PRP on pain score and MOAKS in the included patients with knee $O A$, there were significantly lower mean pain score values when assessed two weeks from the second I.A injection in patients with knee OA, with significant improvement effects on synovitis and bone marrow lesions mainly for mild knee $O A$ and to a lesser extent for moderate cases. Unfortunately, the included treated patients didn't show any significant improvements in the patellafemoral cartilage volume or meniscal disintegrity. In accordance, Burchard et al [32] suggested that intraarticular injection of PRP may improve symptoms of osteoarthritis and decrease pain in patients with knee joint osteoarthritis, independent of the level of cartilage damage quantified by the whole organ MRI scoring method. Furthermore, a study by Laudy et al [33] demonstrated that in patients with knee osteoarthritis, PRP injections resulted in decreased pain, improved function and global assessment, and changes in joint imaging.

Favorable outcome was noticed in all included patients with mild knee $(100 \%)$, while it occurred in $50 \%$ of patients with moderate severity, and (20\%) of patients with severe knee OA showed improvement regarding pain score. In line with our findings, Taniguchi et al [10] reported that intra-articular PRP injection is likely to be a safe treatment option for Japanese patients with mild to moderate osteoarthritis of the knee and may have pain relief for up to 6 months. While PRP therapy has controversially discussed restorative effects on the cartilage, anti-inflammatory effects, down-regulation of cytokine levels and joint homeostasis could explain favorable effects in patients with severe osteoarthritis [34, 35].

Increasing evidence shows that the over-expressed inflammatory cytokines in the inflamed joints play an important pathophysiological role in generating and maintaining OA-induced pain by acting on nociceptive nerve cells [36]. Among the included patients with knee $O A$, there were significantly positive correlations between synovial fluid TNF- $a$ and MIF levels with the pain score. In consistent with our findings, Stannus et al [37] reported that TNF-a was positively linked with total knee worsening and pain.

In conclusion, the current study confirms the presence of inflammatory process involved in the pathogenesis of knee OA including involvement of some inflammatory cytokines in the form of TNF-a and MIF. Additionally, the present study confirms that among the therapeutic mechanisms of using I.A injection of PRP in patients with knee OA is the anti-inflammatory effect, where PRP may lower the synovial fluid cytokines (TNF- $a$ and MIF) with subsequent improvement of pain and synovitis. This favorable outcome is prominently achieved in patients having mild and to a lesser extent moderate $O A$, thus could be established as safe adjuvant biologic therapy for such patients. 


\section{Study Limitation}

Lack of long term follow up of the included patients, which could be approached in future research and relatively small sample size, were the main limitations of the study.

\section{Abbreviations}

OA

Osteoarthritis ; TNF-a:Tumor necrosis factor-a ; MIF:Macrophage migration inhibitory factor ; PRP:Plateletrich plasma ; I.A:intra-articular ; ELISA:enzyme linked immunosorbent assay; BMI:body mass index; VAS:Visual Analog Scale; MOAKS:MRI Osteoarthritis Knee Score.

\section{Declarations}

\section{Acknowledgment}

Not applicable.

\section{Authors' contributions}

Study concept and design: MHH and ES; Clinical evaluation of the cases: ES and HT; IA injection of PRP: ES and HT; Radiological evaluation of patients: GMA-R; Literature research: MHH,SA, NAZ, SYS and THS; Biochemical and laboratory assays: MHH; PRP preparations: $\mathrm{MHH}$ and NAZ; statistical analysis: $\mathrm{MHH}$, SYS, SA and NAZ; drafting the manuscript: MHH; All authors revised and approved the final version of the manuscript.

\section{Funding}

The current research was partially funded by South Valley University, faculty of Medicine, Qena, Egypt.

\section{Availability of data and materials}

The datasets used and/or analyzed during the current study are available from the corresponding author on reasonable request.

\section{Ethical approval and consent to participate}

Prior to start in the study, approval from the Ethics Committee of Faculty of Medicine, South Valley University, Qena, Egypt, was obtained. The ethical approval code (SVU-MED-MBC004-19-1). The study was carried out in accordance with the Declaration of Helsinki. A written informed consent was taken from every included patient.

\section{Consent for publication}


Not applicable

\section{Competing interests}

The author(s) declared no potential conflicts of interest with respect to the research, authorship, and/or publication of this article.

\section{References}

1. Kennedy MI, Whitney K, Evans T, LaPrade RF. Platelet-Rich Plasma and Cartilage Repair. Curr Rev Musculoskelet Med. 2018;11(4):573-82.

2. Richebé P, Capdevila X, Rivat C. Persistent Postsurgical Pain: Pathophysiology and Preventative Pharmacologic Considerations. Anesthesiology. 2018;129(3):590-607.

3. Ayhan E, Kesmezacar H, Akgun I. Intraarticular injections (corticosteroid, hyaluronic acid, platelet rich plasma) for the knee osteoarthritis. World J Orthop. 2014;5(3):351-61.

4. Li H, Xie S, Qi Y, Li H, Zhang R, Lian Y. TNF-a increases the expression of inflammatory factors in synovial fibroblasts by inhibiting the PI3K/AKT pathway in a rat model of monosodium iodoacetateinduced osteoarthritis. Exp Ther Med. 2018;16(6):4737-44.

5. Llamas-Covarrubias MA, Valle Y, Bucala R, Navarro-Hernández RE, Palafox-Sánchez CA, PadillaGutiérrez JR, et al. Macrophage migration inhibitory factor (MIF): genetic evidence for participation in early onset and early stage rheumatoid arthritis. Cytokine. 2013;61(3):759-65.

6. Sampson S, Reed M, Silvers H, Meng M, Mandelbaum B. Injection of platelet-rich plasma in patients with primary and secondary knee osteoarthritis: a pilot study. Am J Phys Med Rehabil. 2010;89:9619.

7. Halpern B, Chaudhury S, Rodeo SA, Hayter C, Bogner E, Potter HG, Nguyen J. Clinical and MRI outcomes after platelet-rich plasma treatment for knee osteoarthritis. Clin J Sport Med. 2013;23:238-9.

8. Nurden AT. Platelets, inflammation and tissue regeneration. Thromb Haemost. 2011;105:13-33.

9. Chakkalakal JV, Jones KM, Basson MA, Brack AS. The aged niche disrupts muscle stem cell quiescence. Nature. 2012;490:355-60.

10. Taniguchi Y, Yoshioka T, Kanamori A, Aoto K, Sugaya H, Yamazaki M. Intra-articular platelet-rich plasma (PRP) injections for treating knee pain associated with osteoarthritis of the knee in the Japanese population: a phase I and Ila clinical trial. Nagoya J Med Sci. 2018;80(1):39-51.

11. Dwyer JT, Melanson KJ, Sriprachy-anunt U, Cross P, Wilson M. Dietary Treatment of Obesity. In: Feingold KR, Anawalt B, Boyce A, et al., eds. Endotext. South Dartmouth (MA): MDText.com, Inc.; 2000.

12. Katz J, Melzack R. Measurement of pain. Surg Clin North Am. 1999;79:231-52.

13. Rogers J, Dieppe P. Paleopathology of osteoarthritis. In: Brandt KD, Doherty M, Lohmander LS, editors. Osteoarthritis. 2nd ed. Oxford: Oxford Univ Press; 2003. pp. 57-65. 
14. Pritzker KP, Gay S, Jimenez SA, Ostergaard K, Pelletier J-P, Revell PA, et al. Osteoarthritis cartilage histopathology: grading and staging. Osteoarthritis Cartilage. 2006;14(1):13-29.

15. Hunter DJ, Guermazi A, Lo GH, Grainger AJ, Conaghan PG, Boudreau RM, et al. Evolution of semiquantitative whole joint assessment of knee OA: MOAKS (MRI Osteoarthritis Knee Score). Osteoarthritis Cartilage. 2011 Aug;19(8):990-1002.

16. Conrozier T, Eymard F, Chouk M, Chevalier X. Impact of obesity, structural severity and their combination on the efficacy of viscosupplementation in patients with knee osteoarthritis. BMC Musculoskelet Disord. 2019;20(1):376.

17. Powell A, Teichtahl AJ, Wluka AE, Cicuttini FM. Obesity: a preventable risk factor for large joint osteoarthritis which may act through biomechanical factors. Br J Sports Med. 2005;39(1):4-5.

18. Felson DT. The epidemiology of knee osteoarthritis: results from the Framingham Osteoarthritis Study. Semin Arthritis Rheum. 1990;20:42-50.

19. Spector TD, Hart DJ, Doyle DV. Incidence and progression of osteoarthritis in women with unilateral knee disease in the general population: the effect of obesity. Ann Rheum Dis. 1994;53:565-8.

20. Snijders GF, den Broeder AA, van Riel PLCM, Straten VHHP, de Man FHR, van den Hoogen FHJ, van den Ende CHM, on behalf of the NOAC study group. Evidence-based tailored conservative treatment of knee and hip osteoarthritis: between knowing and doing. Scand J Rheumatol. 2011;40:225-31.

21. Hawamdeh MZ, Al-Ajlouni JM. The clinical pattern of knee osteoarthritis in Jordan: a hospital based study. Int J Med Sci. 2013;10(6):790-5.

22. Zheng $\mathrm{H}$, Chen $\mathrm{C}$. Body mass index and risk of knee osteoarthritis: systematic review and metaanalysis of prospective studies. BMJ Open. 2015;5(12):e007568.

23. Wallace IJ, Worthington S, Felson DT, Jurmain RD, Wren KT, Maijanen $H$, et al. Knee osteoarthritis has doubled in prevalence since the mid-20th century. Proc Natl Acad Sci U S A. 2017;114(35):9332-6.

24. Hame SL, Alexander RA. Knee osteoarthritis in women. Curr Rev Musculoskelet Med. 2013;6(2):1827.

25. Huang G, Hua S, Yang T, Ma J, Yu W, Chen X. Platelet-rich plasma shows beneficial effects for patients with knee osteoarthritis by suppressing inflammatory factors. Exp Ther Med. 2018;15(3):3096-102.

26. O'Connell B, Wragg NM, Wilson SL. The use of PRP injections in the management of knee osteoarthritis. Cell Tissue Res. 2019;376(2):143-52.

27. Chiu WC, Lai YP, Chou MY. Humanization and characterization of an anti-human TNF-a murine monoclonal antibody. PLoS One. 2011;6:e16373.

28. Kapoor M, Martel-Pelletier J, Lajeunesse D, Pelletier J-P, Fahmi H. Role of proinflammatory cytokines in the pathophysiology of osteoarthritis. Nat Rev Rheumatol. 2011;7:33-42.

29. Lebouvier A, Poignard A, Cavet M, Amiaud J, Leotot J, Hernigou P, et al. Development of a simple procedure for the treatment of femoral head osteonecrosis with intra-osseous injection of bone 
marrow mesenchymal stromal cells: Study of their biodistribution in the early time points after injection. Stem Cell Res Ther. 2015;6:68.

30. Zhang PL, Liu J, Xu L, Sun Y, Sun XC. Synovial Fluid Macrophage Migration Inhibitory Factor Levels Correlate with Severity of Self-Reported Pain in Knee Osteoarthritis Patients. Med Sci Monit. 2016;22:2182-6.

31. Leech M, Metz C, Hall P, Hutchinson P, Gianis K, Smith M, et al. Macrophage migration inhibitory factor in rheumatoid arthritis: evidence of proinflammatory function and regulation by glucocorticoids. Arthritis Rheum. 1999;42(8):1601-8.

32. Burchard R, Huflage $H$, Soost $C$, Richter $O$, Bouillon B, Graw JA. Efficiency of platelet-rich plasma therapy in knee osteoarthritis does not depend on level of cartilage damage. J Orthop Surg Res. 2019;14(1):153.

33. Laudy AB, Bakker EW, Rekers M, Moen MH. Efficacy of platelet-rich plasma injections in osteoarthritis of the knee: A systematic review and meta-analysis. Br J Sports Med. 2015;49:657-72.

34. Kon E, Buda R, Filardo G, Di Martino A, Timoncini A, Cenacchi A, et al. Platelet-rich plasma: intraarticular knee injections produced favorable results on degenerative cartilage lesions. Knee Surg Sports Traumatol Arthrosc. 2010;18(4):472-9.

35. Marmotti A, Rossi R, Castoldi F, Roveda E, Michielon G, Peretti GM. PRP and articular cartilage: a clinical update. Biomed Res Int. 2015;2015:542502.

36. Penninx BW, Abbas H, Ambrosius W, Nicklas BJ, Davis C, Messier SP, et al. Inflammatory markers and physical function among older adults with knee osteoarthritis. J Rheumatol. 2004;31:2027-31.

37. Stannus OP, Jones G, Blizzard L, Cicuttini FM, Ding C. Associations between serum levels of inflammatory markers and change in knee pain over 5 years in older adults: a prospective cohort study. Ann Rheum Dis. 2013;72(4):535-40.

\section{Tables}

Table 1. Comparison of serial synovial fluid levels of TNF-a as regards time of intra-articular PRP injection in patients with knee OA

\begin{tabular}{|c|c|c|c|c|c|c|}
\hline \multirow[b]{2}{*}{$\begin{array}{l}\text { TNF-a }(\mathrm{pg} / \mathrm{mL} \text {, Mean } \\
\pm \mathrm{SD})\end{array}$} & \multirow{2}{*}{$\begin{array}{l}\text { S1 } \\
(n=30)\end{array}$} & \multirow{2}{*}{$\begin{array}{l}S 2 \\
(n=30)\end{array}$} & \multirow{2}{*}{$\begin{array}{l}\text { S3 } \\
(n=30)\end{array}$} & \multicolumn{3}{|c|}{ P-value } \\
\hline & & & & P1 & $\mathrm{P} 2$ & P3 \\
\hline Mild OA & $35.5 \pm 7.4$ & $17.8 \pm 7.5$ & $9.3 \pm 5.4$ & $<.001$ & $\stackrel{<}{0.001}$ & 0.01 \\
\hline Moderate OA & $97.3 \pm 31.7$ & $\begin{array}{l}64.6 \pm \\
15.3\end{array}$ & $\begin{array}{l}44.8 \pm \\
11.5\end{array}$ & 0.002 & $\hat{0}_{0.001}^{<}$ & 0.048 \\
\hline Severe OA & $\begin{array}{l}144.8 \pm \\
13.6\end{array}$ & $82.4 \pm 4.3$ & $59.7 \pm 3.9$ & $<.001$ & $<.001$ & $<.001$ \\
\hline
\end{tabular}


$\mathrm{P} 1=\mathrm{S} 1$ vs. S2; P2= S1 vs. S3; P3= S2 vs. S1.

N.B: TNF-a: tumor necrosis factor-a; PRP: platelet rich plasma; OA: osteoarthritis; SD: standard deviation; S1: first synovial fluid sample assay (before IA injection of PRP); S2: second synovial fluid sample assay (after 2 weeks of the $1^{\text {st }}$ IA injection of PRP); S3: third synovial fluid sample assay (after 2 weeks of the $2^{\text {nd }}$ IA injection of PRP).

Table 2. Comparison of serial synovial fluid levels of MIF as regards time of intra-articular PRP injection in patients with knee $\mathrm{OA}$

\begin{tabular}{|c|c|c|c|c|c|c|}
\hline \multirow[b]{2}{*}{ MIF ( $\mu \mathrm{g} / \mathrm{l}$, , Mean $\pm S D)$} & \multirow{2}{*}{$\begin{array}{l}\text { S1 } \\
(n=30)\end{array}$} & \multirow{2}{*}{$\begin{array}{l}S 2 \\
(n=30)\end{array}$} & \multirow{2}{*}{$\begin{array}{l}\text { S3 } \\
(n=30)\end{array}$} & \multicolumn{3}{|l|}{ P-value } \\
\hline & & & & P1 & P2 & P3 \\
\hline Mild OA & $7.7 \pm 1.5$ & $4.9 \pm 0.9$ & $1.25 \pm 0.9$ & $<0.001$ & $<0.001$ & $<0.001$ \\
\hline Moderate OA & $47.8 \pm 14.9$ & $25.3 \pm 7.9$ & $10.6 \pm 2.6$ & $<0.001$ & $<0.001$ & 0.003 \\
\hline Severe OA & $315.1 \pm 23.2$ & $107.7 \pm 12.3$ & $82.9 \pm 8.2$ & $<0.001$ & $<0.001$ & 0.002 \\
\hline
\end{tabular}

$$
\mathrm{P} 1=\mathrm{S} 1 \text { vs. S2; P2= S1 vs. S3; P3= S2 vs. S1. }
$$

N.B: MIF: Macrophage migration inhibitory factor; PRP: platelet rich plasma; OA: osteoarthritis; SD: standard deviation; S1: first synovial fluid sample assay (before IA injection of PRP); S2: second synovial fluid sample assay (after 2 weeks of the $1^{\text {st }}$ IA injection of PRP); S3: third synovial fluid sample assay (after 2 weeks of the $2^{\text {nd }}$ IA injection of PRP).

Table 3. Effect of intra-articular injection of platelet rich plasma (PRP) on pain score among patients with knee osteoarthritis (OA) of various severities

\begin{tabular}{|llll|}
\hline Patients groups & \multicolumn{2}{l|}{ Pain score $($ Mean \pm SD $)$} & P-value \\
\cline { 2 - 3 } & Pre- injection & 2 weeks from the second injection & \\
\hline Mild OA $(\mathbf{n = 3 0})$ & $3.1 \pm 0.9$ & $1.6 \pm 0.5$ & 0.001 \\
\hline Moderate OA $(\mathbf{n}=\mathbf{3 0})$ & $5.9 \pm 0.9$ & $2.4 \pm 1.9$ & 0.002 \\
\hline Severe OA $(\mathbf{n}=\mathbf{3 0})$ & $8.7 \pm 0.7$ & $5.1 \pm 1.9$ & $<0.001$ \\
\hline
\end{tabular}




\begin{tabular}{|c|c|c|c|}
\hline \multirow[t]{2}{*}{ Patients groups } & \multicolumn{2}{|c|}{$\begin{array}{l}\text { Patello-femoral cartilage volume } \\
(\text { Mean } \pm S D)\end{array}$} & \multirow[t]{2}{*}{ P-value } \\
\hline & Pre- injection & 2 weeks from the second I.A injection & \\
\hline Mild OA $(n=30)$ & $0.24 \pm 0.04$ & $0.22 \pm 0.05$ & 0.227 \\
\hline Moderate OA $(n=30)$ & $0.55 \pm 0.10$ & $0.55 \pm 0.10$ & 1.0 \\
\hline Severe OA $(n=30)$ & $0.71 \pm 0.08$ & $0.70 \pm 0.08$ & 0.677 \\
\hline
\end{tabular}

\begin{tabular}{|lrrl|}
\hline & \multicolumn{3}{c|}{ Meniscal desintegrity (Mean \pm SD) } \\
\hline Mild OA $(\mathbf{n}=\mathbf{3 0})$ & $1.0 \pm 0.0$ & $1.0 \pm 0.0$ & - \\
\hline Moderate OA $(\mathbf{n}=\mathbf{3 0})$ & $2.0 \pm 0.0$ & $2.0 \pm 0.0$ & -- \\
\hline Severe OA $(\mathbf{n}=\mathbf{3 0})$ & $3.0 \pm 0.0$ & $2.1 \pm 1.4$ & 0.280 \\
\hline
\end{tabular}

\begin{tabular}{|lccc|}
\hline & \multicolumn{3}{c|}{ Bone marrow abnormality (Mean \pm SD) } \\
\hline Mild OA & $0.30 \pm 0.02$ & $0.19 \pm 0.07$ & 0.001 \\
\hline Moderate OA & $0.45 \pm 0.13$ & $0.41 \pm 0.18$ & 0.684 \\
\hline Severe OA & $0.75 \pm 0.06$ & $0.73 \pm 0.06$ & 0.579 \\
\hline
\end{tabular}

\begin{tabular}{|lccl|}
\hline \multicolumn{4}{|c|}{ Synovitis (Mean \pm SD) } \\
\hline Mild OA & $0.80 \pm 0.79$ & $0.02 \pm 0.01$ & 0.0393 \\
\hline Moderate OA & $1.80 \pm 0.91$ & $0.03 \pm 0.0$ & 0.001 \\
\hline Severe OA & $1.81 \pm 1.54$ & $0.03 \pm 0.0$ & 0.143 \\
\hline
\end{tabular}

N.B: I.A (intra-articular); OA (osteoarthritis); PRP (platelet-rich plasma); MRI Osteoarthritis Knee Score (MOAKS); SD: standard deviation.

Table 5. Comparison of the therapeutic outcomes of the IA injection of the PRP in patients with various severities of knee OA 


\begin{tabular}{|c|c|c|c|c|c|c|c|c|c|c|}
\hline \multirow[b]{3}{*}{ Outcome } & \multirow[b]{3}{*}{$\begin{array}{l}\text { Improved } \\
\text { (No.,\%) }\end{array}$} & \multirow{2}{*}{\multicolumn{2}{|c|}{$\begin{array}{l}\text { Mild OA } \\
(n=30)\end{array}$}} & \multirow{2}{*}{\multicolumn{2}{|c|}{$\begin{array}{l}\text { Moderate OA } \\
(n=30)\end{array}$}} & \multirow{2}{*}{\multicolumn{2}{|c|}{$\begin{array}{l}\text { Severe OA } \\
(n=30)\end{array}$}} & \multicolumn{3}{|c|}{ P-value } \\
\hline & & & & & & & & \multirow{2}{*}{$\begin{array}{l}\text { P1 } \\
0.036\end{array}$} & \multirow{2}{*}{$\begin{array}{l}\text { P2 } \\
0.001\end{array}$} & \multirow{2}{*}{$\begin{array}{l}\text { P3 } \\
0.175\end{array}$} \\
\hline & & 30 & $100 \%$ & 15 & $50 \%$ & 6 & $20 \%$ & & & \\
\hline & $\begin{array}{l}\text { Not improved } \\
\text { (No.,\%) }\end{array}$ & 0 & $0 \%$ & 15 & $50 \%$ & 24 & $80 \%$ & & & \\
\hline
\end{tabular}

Figures 

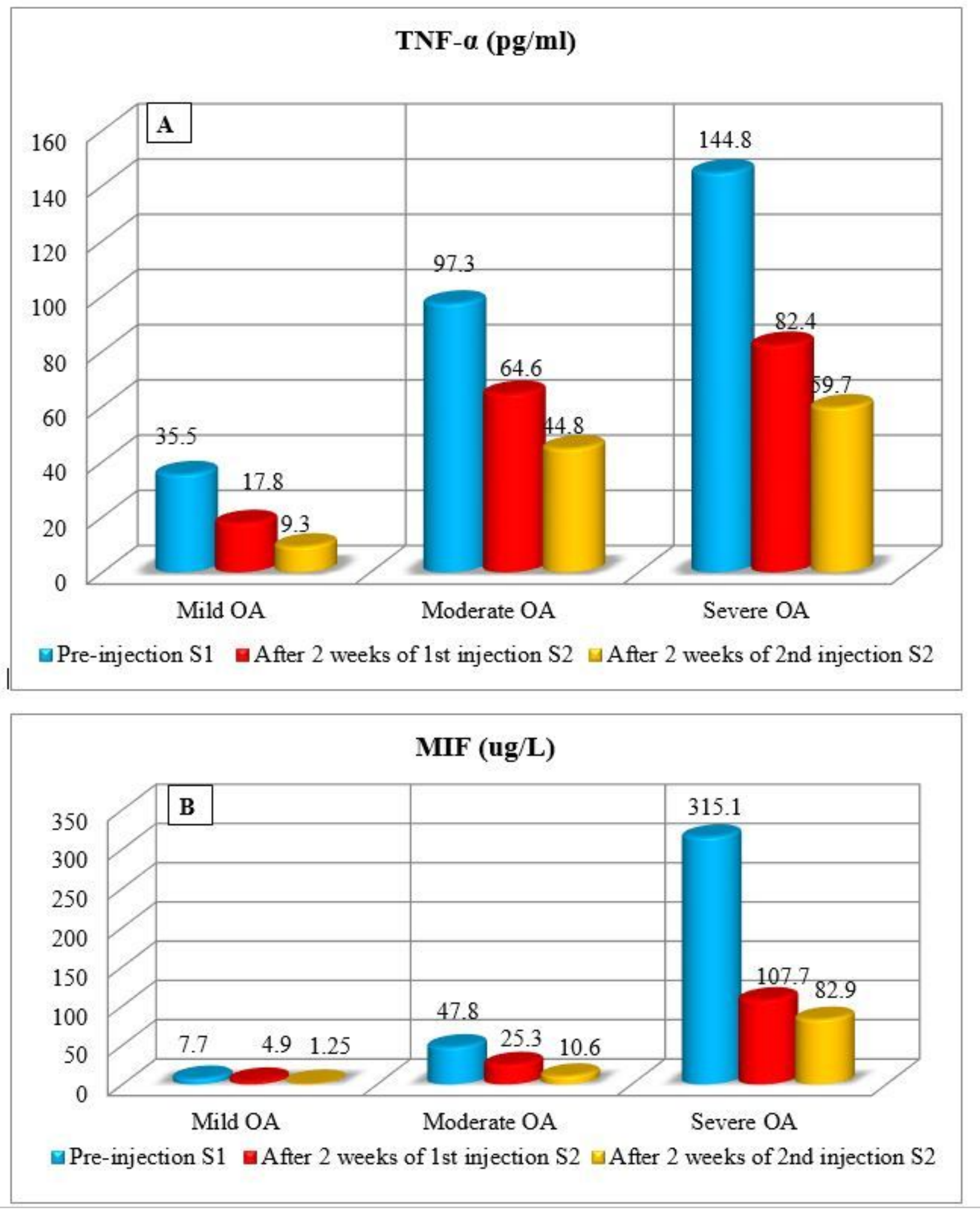

\section{Figure 1}

Comparison of serial synovial fluid levels of tumor necrosis factor (TNF) a (AA) and macrophage migration inhibitory factor (MIF) (B) as regards severity of knee osteoarthritis (OA) 


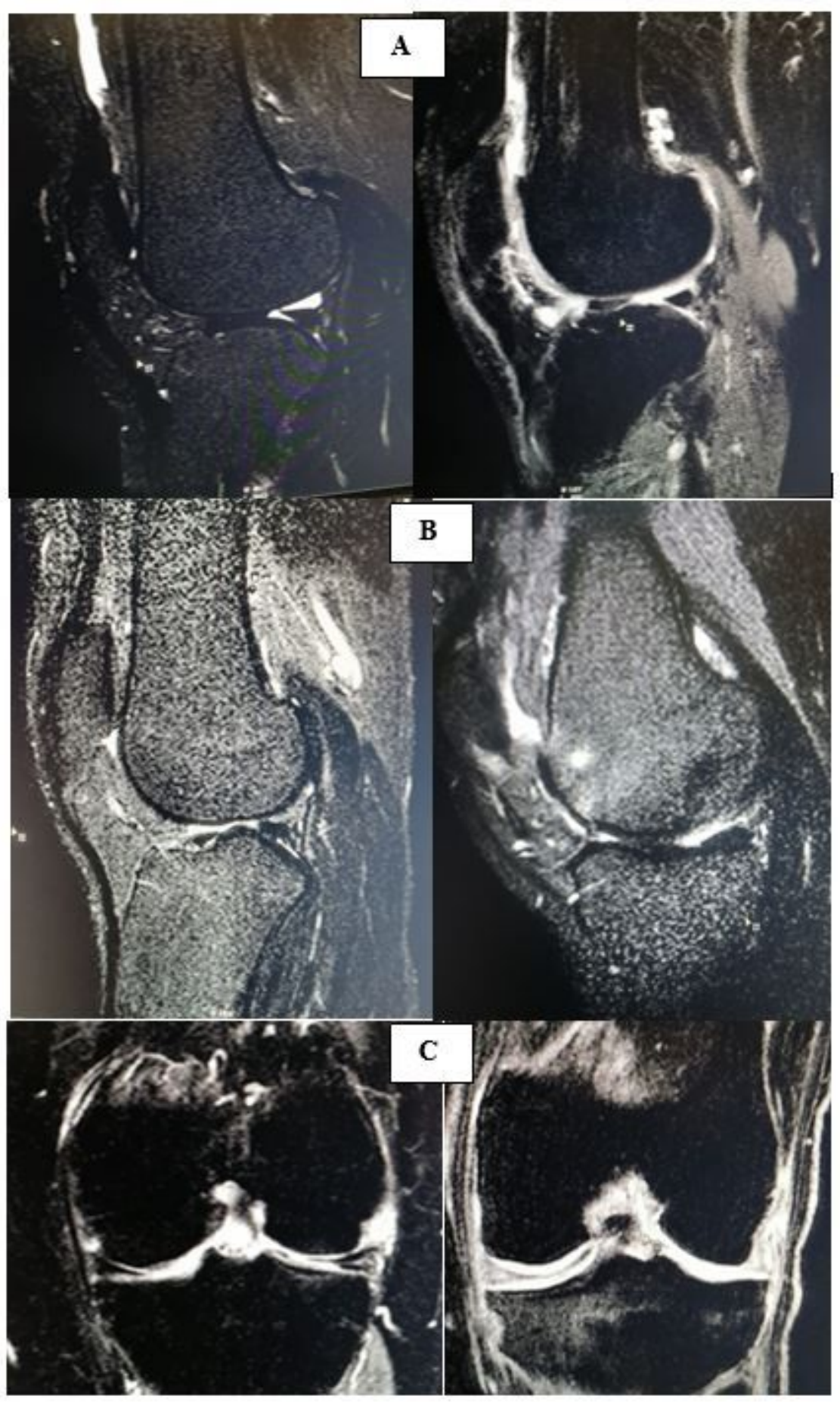

Before

After

Figure 2

MRI analysis of knee joint before and after IA injection of PRP two weeks after the second injection, MRI signals showed that there were significant improvement in the synovitis (A) and bone marrow lesions (B) with no improvement in cartilage loss(C) compared with pre injection condition. 

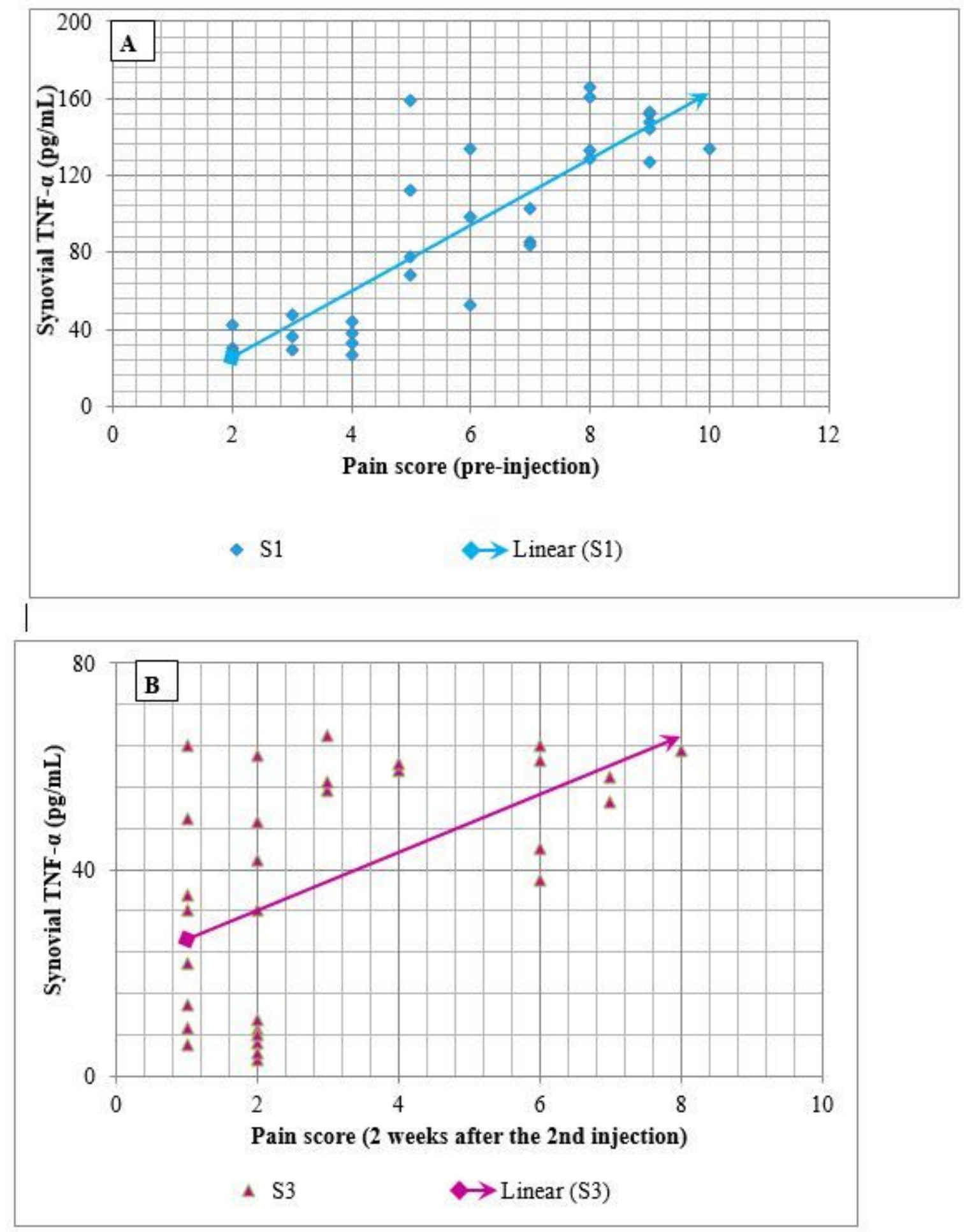

Figure 3

Correlations of pain score with serial synovial TNF-a levels among patients with knee osteoarthritis both pre-injection (A) and 2 weeks after the 2 nd injection (B). 

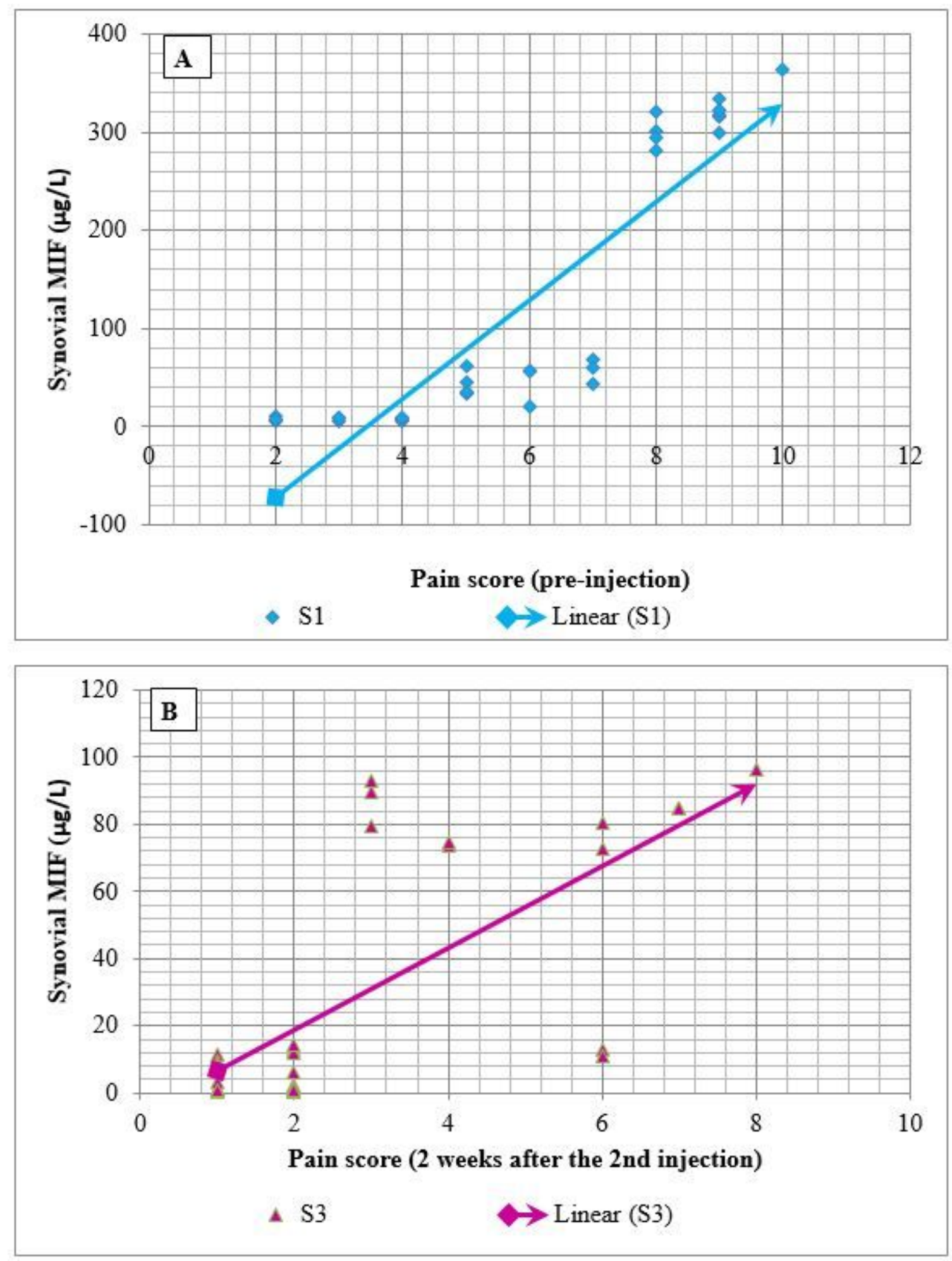

\section{Figure 4}

Correlations of pain score with serial synovial MIF levels among patients with knee osteoarthritis both pre-injection (A) and 2 weeks after the 2nd injection (B). 